\title{
Interrelation between plasma membrane calcium ATPase-1 and inflammatory mediators expression in gingival tissue depending on severity of periodontitis
}

\author{
Da-Le Yoon ${ }^{1}$, Su-Yeon Park², Yong-Gun $\mathrm{Kim}^{3}$, and Jae-Mok Lee ${ }^{4 *}$ \\ ${ }^{1}$ Doctor of Dental Clinic, Department of Periodontics, School of Dentistry, Kyungpook National University, Daegu, Republic of Korea \\ ${ }^{2}$ Resident, Department of Periodontics, School of Dentistry, Kyungpook National University, Daegu, Republic of Korea \\ ${ }^{3}$ Associate Professor, Department of Periodontics, School of Dentistry, Kyungpook National University, Daegu, Republic of Korea \\ ${ }^{4}$ Professor, Department of Periodontics, School of Dentistry, Kyungpook National University, Daegu, Republic of Korea
}

\begin{abstract}
We investigated the effects of severity of periodontitis on inflammation progression by observing the expression and interaction of inflammatory mediators such as plasma membrane calcium ATPase (PMCA)-1, interleukin (IL)-11, and leukemia inhibitory factor (LIF), which are involved in bone metabolism, in healthy tissues and tissues according to the severity of inflammation. The aim of this study was to investigate the effect of these factors on periodontal tissue destruction. Gingival tissue specimens were collected from patients with periodontal disease and healthy individuals, at the Department of Periodontics, Kyungpook National University Dental Hospital. Specimen was recruited from the patients were obtained during periodontal surgery. Western blotting was used to observe the expression of PMCA-1, IL-11, and LIF in each tissue. The expression of PMCA-1 was increased in periodontitis groups and was highest in severe periodontitis group. IL-11 expression was increased in periodontitis groups and was highest in severe periodontitis group. LIF expression was decreased in periodontitis groups and was highest in normal group. There were a statistically significant difference between normal and moderate group, normal and severe periodontitis group, and moderate and severe periodontitis group. In conclusion, PMCA-1, IL-11, and LIF appear to be associated with the severity of chronic periodontitis. These factors may influence each other.
\end{abstract}

Key Words: Chronic periodontitis, Inflammation, Interleukin, Leukemia inhibitory factor, Plasma membrane calcium ATPase

(c) This is an open-access article distributed under the terms of the Creative Commons Attribution Non-Commercial License (http://creativecommons.org/licenses/by-nc/4.0) which permits unrestricted noncommercial use, distribution, and reproduction in any medium, provided the original work is properly cited.

\section{INTRODUCTION}

Periodontal diseases demonstrate various clinical symptoms such as gingival bleeding, swelling, formation of periodontal pocket along with alveolar bone destruction. Generally, incidence of periodontitis cause the broad extent of periodontium destruction by gingival inflammation resulted from the various local and systemic factors. During this process, many environmental and external conditions are also highly related [1].

Although bacteria that colonize the tooth surface and gingival sulcus are known to initiate the periodontal disease [2], it is believed that during the disease process, host response plays important role in the both connective tissue

Received January 10, 2020; Revised [1] February 14, 2020; [2] February 18, 2020; Accepted February 18, 2020

*Corresponding author: Jae-Mok Lee, Department of Periodontics, School of Dentistry, Kyungpook National University, 2175 Dalgubeol-daero, Jung-gu, Daegu 41940, Republic of Korea.

Tel: +82-53-600-7522, Fax: +82-53-427-3263, E-mail: leejm@knu.ac.kr

Copyright $\odot$ 2020, Oral Biology Research Institute 
and bone destruction which is the key features of the disease. Cytokine stimulates inflammatory events that activate effector mechanism, and as inflammatory mediators between bacterial stimulation and tissue destruction, it plays important role in various cells that promotes or inhibits the process of periodontitis [3]. Thus, there are many studies investigating the effect of cytokine on the target cell. Since osteoclast, one of target cells under the effect of cytokine, is known to cause the alveolar bone resorption and determines the severity of periodontitis, it is very important to figure out the factors regulating its activation.

Plasma membrane calcium ATPase (PMCAs) which is recently known as a factor related to the regulation of osteoclast. It is one of P-type pump family, and during the reaction cycle it forms the high-energy phosphorylated intermediate [4]. Accurate regulation of $\mathrm{Ca}^{2+}$ dynamics is critical for proper differentiation and function of osteoclasts. There are 4 major types of PMCAs isoform and they are expressed in different parts of the human body $[5,6]$. Among these isoforms, PMCA-1 is known to play important role in osteoclastogenesis and bone homeostasis regulation by regulating $\mathrm{Ca}^{2+}$ signaling of osteoclast. In vitro study, PMCAs affect the differentiation of osteoclast by inhibiting receptor activator of NF-kappa B ligand (RANKL)-induced $\mathrm{Ca}^{2+}$ shift in immature/undifferentiated cell. Furthermore, increased expression of PMCAs in mature osteoclasts prevents osteoclast apoptosis both in vitro and in vivo study. Based on such study result, 'dual role' of PMCAs toward the osteoclastogenesis has been suggested [7]. Though it is expected to play significant role in the regulation of osteoclast, not many researches on this subject related to the periodontitis are available. Therefore, there is need for study investigating how the expression of PMCA-1 is correlated with periodontitis.

Interleukin (IL)-11 is a human protein which arises from the bone marrow-derived fibroblast or stromal cells and it first separated from the stromal cells. IL-11 is key regulator of multiple events in hematopoiesis, most notably the stimulation of megakaryocyte maturation [8]. At the same time, supporting the human osteoclast formation through RANKL-independent pathway is also well known function of IL-11 [9]. According to Martuscelli et al. [10], after the systemic injection of IL-11 to ligature induced periodontal disease model in beagle dog, significant improvement in periodontal attachment loss has been reported. However, Becerik et al. [11] report that increase of IL-11 demonstrates positive correlation with pocket depth (PD), clinical attachment level (CAL), and bleeding on probing (BOP) on gingival crevicular fluid (GCF) level. Due to such conflicting results of former studies, there is controversy on the role of IL-11. Thus, investigating expression of IL-11 in gingival tissue depending on the severity would be helpful to elucidate the role of IL-11 in periodontitis.

Leukemia inhibitory factor (LIF) is mainly expressed from the trophectoderm of the developing embryo. LIF affects cell growth by inhibition of cell differentiation [12]. In addition, it has been clearly demonstrated that LIF is also associated with bone resorption through RANKL-dependent pathway [13]. Becerik et al. [11] report lower LIF level in GCF with chronic periodontitis. In addition, it shows significant positive correlation with GCF IL-11 level observed in the same study. LIF which clearly has effect on the differentiation and growth of cells might have possible correlation with periodontitis since various types of cells are involved in the progression and severity of periodontitis.

The most important feature of the periodontitis is the destruction of alveolar bone following the attachment loss and osteoclast plays important role during the process of alveolar bone destruction. In order to play bone resorption role at the inflammatory periodontal tissue, osteoclast should go through several processes including fusion of osteoclast precursor such as formation, differentiation and activation. During the process, complex regulation of stimulation and inhibition becomes accomplished by various factors involved in different pathways [14]. As mentioned above, PMCA-1 plays dual role (suppressing the differentiation through RANKL-dependent pathway in immature cell while maintaining osteoclastic activity by preventing apoptosis in mature cell) and both IL-11 and LIF have function of differentiation stimulus each through RANKLindependent pathway and RANKL-dependent pathway respectively.

Since these three factors have possibility to play certain role in osteoclastic activity, it is also possible that expression of these factors might show different features depending on the osteoclastic activity which is varied according to 
the severity of periodontitis.

\section{MATERIALS AND METHODS}

\section{Study population and tissue sampling}

Gingival tissue specimens were collected from patients with periodontal disease and from healthy individuals, at the Department of Periodontics, Kyungpook National University Dental Hospital. Followings were specified as exclusion criteria: pregnancy, smokers, and any kind of systemic disease that might influence response to treatment such as diabetes mellitus, hypertension, cardiovascular disease, arthritis, liver disease, renal disease, malignant tumor, autoimmune disease, and metabolic bone disease, etc. Specimen was recruited from the patients were obtained during periodontal surgery including surgical crown lengthening or tooth extraction by internal bevel incision. The gingival tissue samples are consisted of junctional and crevicular epithelium and connective tissue. Before any surgical procedures, informed consent was achieved from all the subjects. The diagnosis and classification depending on the severity of chronic periodontitis were established based on the clinical and radiographic criteria. The sulcus bleeding index (SBI) value [15] and probing pocket depth (PPD) [16] were measured to define the clinical periodontal parameters. These clinical parameters were used to determine clinical criteria. From the available radiographic images, level of bone resorption could be observed. 16 patients were subjected in each group, and tissue were collected from the patients.

1) Normal (group 1, control group, $n=16$ ): clinically healthy gingiva without bleeding (SBI 0, 1) that shows no evidence of bone resorption, periodontal pockets or loss of attachment $(\mathrm{PPD} \leq 3 \mathrm{~mm})$

2) Moderate chronic periodontitis (group 2, n=16): chronic periodontitis showing evidence of loss of attachment with bone resorption, $3 \mathrm{~mm}<\mathrm{PPD} \leq 6 \mathrm{~mm}$, and gingival inflammation (SBI 3)

3) Severe chronic periodontitis (group 3, $n=16$ ): chronic periodontitis showing clear evidence of loss of attachment with bone resorption, PPD $>6 \mathrm{~mm}$, and gingival inflammation (SBI 3)

The study protocol was approved by the Ethical Committee of Clinical Experiments, Kyungpook National University Hospital (IRB no. KNUH 2013-05-036-002).

Demographic variable of the patients are shown in Table 1. There are no significant differences in sex and age among each group according to the result came from the chisquare test and one-way ANOVA analysis each performed for the gender and age respectively. After periodontal surgery, tissue specimens were immediately placed on liquid nitrogen and subsequently frozen $\left(-70^{\circ} \mathrm{C}\right)$.

\section{Protein isolation and immunoblotting (Western blot analysis)}

For Western blotting, as previously described [17,18], frozen tissues were mechanically broken down using a homogenizer in RIPA lysis buffer (10 mM EDTA, 0.15M $\mathrm{NaCl}$ ) with 1:30 diluted protease inhibitor cocktail (Roche, Mannheim, Germany) [19]. The lysates were centrifuged at $12,000 \mathrm{~g}$ for $15 \mathrm{~min}$ at $4^{\circ} \mathrm{C}$. Quantitative analysis on protein concentrations of supernatant liquid were performed by a Bradford protein assay (Quick Start; BIO-RAD, Hercules, CA, USA) using bovine serum albumin (BSA) as standard. Lysates were boiled for $3 \mathrm{~min}$ in sodium dodecyl sulfate (SDS) samples buffer (1 M Tris-Cl [pH 6.8], 40\% glycerol, 8\% SDS,

Table 1. Demographic characteristic of the study subjects

\begin{tabular}{lcccc}
\hline Variable & Group 1 (n=16) & Group 2 (n=16) & Group 3 (n=16) & p-value \\
\hline Sex (male/female) & $7 / 9$ & $9 / 7$ & $9 / 7$ & 0.816 \\
Age (y) & $51.31 \pm 10.96$ & $48.13 \pm 7.13$ & $50.44 \pm 6.22$ & 0.542 \\
PD & (from 24 to 68) & $($ from 35 to 64) & (from 38 to 61) & \\
\hline
\end{tabular}

Values are presented as number only or mean \pm standard deviation (range).

Group 1, normal group; group 2, moderate periodontitis group; group 3, severe periodontitis group; PD, pocket depth. 
$2 \%$ mercapto-ethanol, $0.002 \%$ bromophenole blue). Prepared samples were separated by $15 \%$ SDS-polyacrylamide gels using gel electrophoresis, which maintains polypeptides in a denatured state, and transferred to a polyvinylidene difluride membrane to make the proteins accessible to antibody detection. Blocking of the membranes was subsequently achieved in Tris-buffered saline (TBS) with a min percentage of detergent such as Tween 20 (Sigma Aldrich, St. Louis, MO, USA) containing 5\% powdered milk for $1 \mathrm{hr}$. Then, the membranes were incubated with polyclonal antiPMCA-1, anti-IL-11 and anti-LIF antibody (diluted 1:1,000 in TBS; Santa Cruz Biotechnology Inc., Dallas, TX, USA) for overnight at $4^{\circ} \mathrm{C}$. The membranes were washed in TBS with Tween 20 three times for 10 min and incubated with a horseradish peroxidase-linked donkey anti-goat secondary antibody for anti-PMCA-1, anti-IL-11, and anti-LIF antibody (diluted 1:2,000 in TBS) for $1 \mathrm{hr}$ at room temperature. After that, membranes were rinsed three times for $10 \mathrm{~min}$ with Tween 20. The membranes were developed with an ECL Plus development kit (Amsterdam, Beckinghamshire, UK). The quantitative analysis of PMCA-1, IL-11, and LIF along with their $\beta$-actin (Abcam, Cambridge, UK) was performed using a densitometer (Image Gauge V 3.46, Koshin Graphic Systems; Fuji Photo Film Co., Tokyo, Japan). After normalization of each sample, comparative levels of PMCA1 , IL-11, and LIF were expressed as a ratio of PMCA-1, IL11 , and LIF $/ \beta$-actin then the differences among groups were evaluated.

\section{Statistical analysis of the Western blot results}

All data were presented as means \pm standard deviation and results were statistically analyzed via SPSS ver. 23.0 (IBM

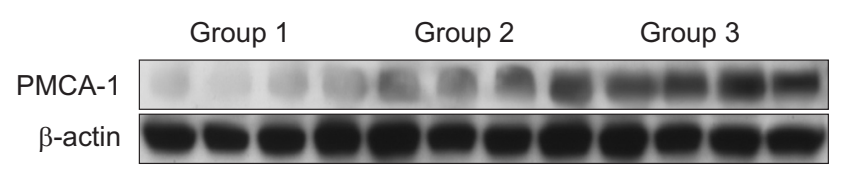

Fig. 1. Western analysis of plasma membrane calcium ATPase (PMCA)-1 showing 4 representative samples in each group. Expression of PMCA-1 shows increasing tendency in group 2 (moderate chronic periodontitis group) and group 3 (severe chronic periodontitis group) comparing to group 1 (normal group). The highest level could be found in group 3 .
Corp., Armonk, NY, USA). The PMCA-1, IL-11, and LIF levels among the different groups were compared using one way ANOVA followed by Dunnett post-hoc test. $p<0.05$ was considered to be statistically significant. The correlations between gingival PMCA-1, IL-11, and LIF levels were evaluated and compared using Spearman correlation coefficients. $p<0.05$ was considered significant.

\section{RESULTS}

The gingival tissue from the patients of normal group (group 1), moderate periodontitis group (group 2), and severe periodontitis group (group 3) showed the expression of PMCA-1 corresponding to molecular weight about $130 \mathrm{kDa}$ in all samples. One representative band of PMCA1 is shown Fig. 1. The expression levels of PMCA-1 were increased in order of group 1, group 2, and group 3. The quantification of PMCA-1 expression is shown in Table 2 and summarized as a graph in Fig. 2. The mean normalized value of PMCA-1 expression relative to its $\beta$-actin was

Table 2. Normalized PMCA-1 expressions by PMCA- $1 / \beta$-actin

\begin{tabular}{cccc}
\hline Sample & Group 1 & Group 2 & Group 3 \\
\hline 1 & 0.060 & 0.258 & 0.296 \\
2 & 0.044 & 0.164 & 0.264 \\
3 & 0.065 & 0.170 & 0.170 \\
4 & 0.114 & 0.213 & 0.213 \\
5 & 0.067 & 0.095 & 0.095 \\
6 & 0.086 & 0.090 & 0.090 \\
7 & 0.064 & 0.093 & 0.093 \\
8 & 0.060 & 0.198 & 0.198 \\
9 & 0.087 & 0.156 & 0.156 \\
10 & 0.138 & 0.242 & 0.242 \\
11 & 0.190 & 0.191 & 0.191 \\
12 & 0.206 & 0.180 & 0.180 \\
13 & 0.118 & 0.109 & 0.109 \\
14 & 0.082 & 0.064 & 0.064 \\
15 & 0.092 & 0.087 & 0.087 \\
16 & 0.088 & 0.154 & 0.154 \\
Mean \pm standard & $0.098 \pm 0.046^{*}$ & $0.154 \pm 0.059^{\dagger}$ & $0.242 \pm 0.107^{\ddagger}$ \\
deviation & & & \\
\hline
\end{tabular}

PMCA, plasma membrane calcium ATPase; group 1, normal group; group 2, moderate periodontitis group; group 3, severe periodontitis group.

${ }^{*}$ Significant difference between group 1 and group $2(p<0.05) .{ }^{\dagger}$ Significant difference between group 2 and group $3(p<0.05)$. ${ }^{*}$ Significant difference between group 1 and group $3(p<0.05)$. 
$0.098 \pm 0.046$ in group $1,0.154 \pm 0.059$ in group $2,0.242 \pm$ 0.107 in group 3. Significant difference could be found between group 1 and group $2(p=0.016$ ), group 2 and group 3 $(p=0.023)$, and group 1 and group $3(p<0.001)(p<0.05)$.

The gingival tissue from the patients of normal group (group 1), moderate periodontitis group (group 2), and severe periodontitis group (group 3) showed the expression of IL-11 corresponding to molecular weight about $20 \mathrm{kDa}$ in all samples. One representative band of IL-11 is shown Fig. 3. The expression levels of IL-11 were increased in order of group 1, group 2, and group 3 .

The quantification of IL-11 expression is shown in Table 3 and summarized as a graph in Fig. 4. The mean normalized value of IL-11 expression relative to its $\beta$-actin was $0.096 \pm 0.047$ in group $1,0.208 \pm 0.092$ in group $2,0.344 \pm$

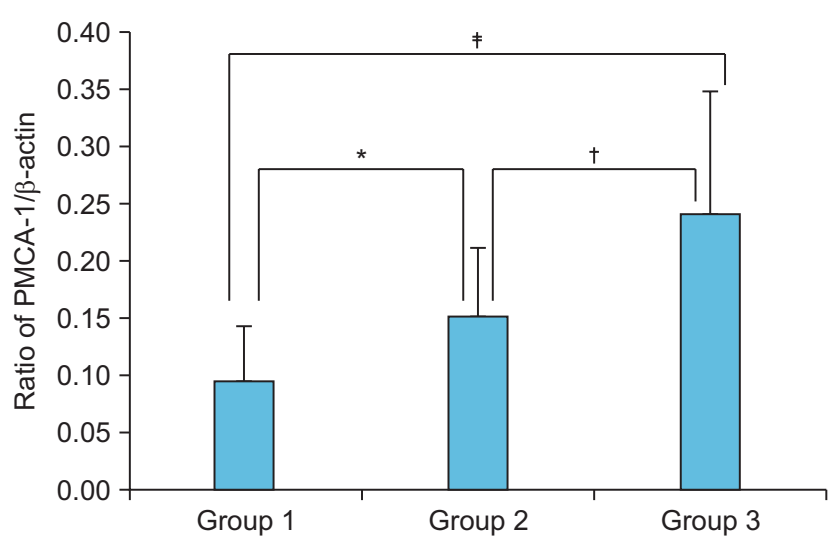

Fig. 2. Graph of normalized plasma membrane calcium ATPase (PMCA)-1 expressions by PMCA- $1 / \beta$-actin. The average amounts and standard deviation of PMCA-1 level (ratio of PMCA- $1 / \beta$-actin) in group 1 (normal group), group 2 (moderate chronic periodontitis group), and group 3 (severe chronic periodontitis group) were used in this graph. The levels of PMCA-1 in the inflamed gingiva were higher than those of healthy gingiva. ${ }^{*}$ Significant difference between group 1 and group $2(p=0.016)$. ${ }^{\dagger}$ Significant difference between group 2 and group $3(p=0.023)$. ${ }^{\ddagger}$ Significant difference between group 1 and group $3(p<0.001)$.

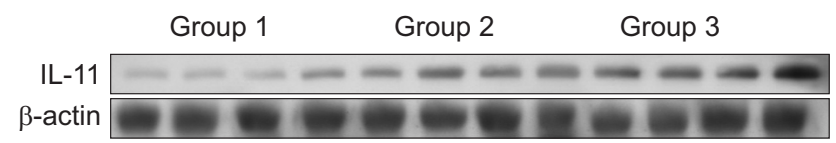

Fig. 3. Western analysis of interleukin (IL)-11 showing 4 representative samples in each group. Expression of IL-11 shows increasing tendency in group 2 (moderate chronic periodontitis group) and group 3 (severe chronic periodontitis group) comparing to group 1 (normal group). The highest level could be found in group 3 .
0.122 in group 3. Significant difference could be found between group 1 and group $2(p=0.001)$, group 2 and group 3 $(p=0.004)$, and group 1 and group $3(p<0.001)(p<0.05)$.

Table 3. Normalized IL-11 expressions by IL-11/ $\beta$-actin

\begin{tabular}{cccc}
\hline Sample & Group 1 & Group 2 & Group 3 \\
\hline 1 & 0.034 & 0.255 & 0.334 \\
2 & 0.094 & 0.277 & 0.399 \\
3 & 0.160 & 0.350 & 0.417 \\
4 & 0.190 & 0.374 & 0.478 \\
5 & 0.088 & 0.079 & 0.185 \\
6 & 0.087 & 0.187 & 0.222 \\
7 & 0.066 & 0.157 & 0.247 \\
8 & 0.089 & 0.142 & 0.467 \\
9 & 0.098 & 0.184 & 0.391 \\
10 & 0.078 & 0.147 & 0.259 \\
11 & 0.053 & 0.236 & 0.445 \\
12 & 0.188 & 0.283 & 0.579 \\
13 & 0.099 & 0.055 & 0.197 \\
14 & 0.053 & 0.127 & 0.159 \\
15 & 0.047 & 0.183 & 0.364 \\
16 & 0.109 & 0.294 & 0.367 \\
Mean \pm standard & $0.096 \pm 0.047^{*}$ & $0.208 \pm 0.092^{\dagger}$ & $0.344 \pm 0.122^{\ddagger}$ \\
deviation & & & \\
\hline
\end{tabular}

IL, interleukin; group 1, normal group; group 2, moderate periodontitis group; group 3 , severe periodontitis group.

${ }^{*}$ Significant difference between group 1 and group $2(p<0.05) .{ }^{\dagger} \mathrm{Sig}-$ nificant difference between group 2 and group $3(p<0.05)$. "Significant difference between group 1 and group $3(p<0.05)$.

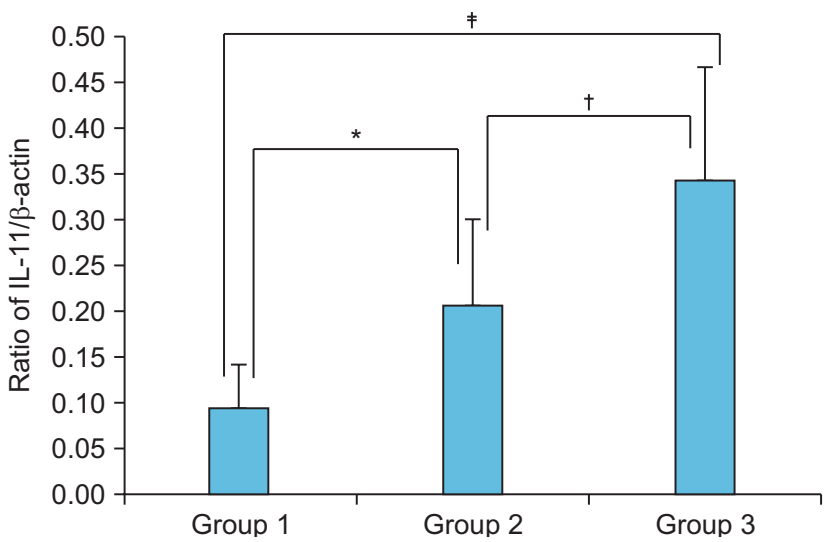

Fig. 4. Graph of normalized interleukin (IL)-11 expressions by IL-11/ $\beta$-actin. The average amounts and standard deviation of IL-11 level (ratio of IL-11/ $\beta$-actin) in group 1 (normal group), group 2 (moderate chronic periodontitis group), and group 3 (severe chronic periodontitis group) were used in this graph. The levels of IL-11 in the inflamed gingiva were higher than those of healthy gingiva. ${ }^{*}$ Significant difference between group 1 and group $2(p<0.001)$. ${ }^{\dagger}$ Significant difference between group 2 and group $3(p=0.004)$. " Significant difference between group 1 and group $3(p<0.001)$. 
The gingival tissue from the patients of normal group (group 1), moderate periodontitis group (group 2), and severe periodontitis group (group 3) showed the expression of LIF corresponding to molecular weight about $20 \mathrm{kDa}$ in all samples. One representative band of LIF is shown Fig. 5. The expression levels of LIF were decreased in order of group 1, group 2, and group 3 .

The quantification of LIF expression is shown in Table 4 and summarized as a graph in Fig. 6. The mean normalized value of LIF expression relative to its $\beta$-actin was $0.428 \pm$ 0.127 in group $1,0.186 \pm 0.073$ in group $2,0.054 \pm 0.027$ in group 3. Significant difference could be found between group 1 and group $2(p<0.001)$, group 2 and group 3 ( $p$

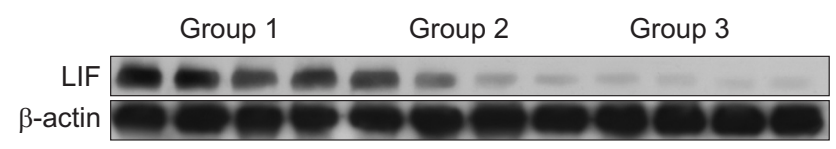

Fig. 5. Western analysis of leukemia inhibitory factor (LIF) showing 4 representative samples in each group. Expression of LIF levels shows decreasing tendency in group 2 (moderate chronic periodontitis group) and group 3 (severe chronic periodontitis group) comparing to group 1 (normal group). The highest level could be found in group 1 .

Table 4. Normalized LIF expressions by LIF/ $\beta$-actin

\begin{tabular}{cccc}
\hline Sample & Group 1 & Group 2 & Group 3 \\
\hline 1 & 0.559 & 0.274 & 0.084 \\
2 & 0.453 & 0.182 & 0.067 \\
3 & 0.360 & 0.158 & 0.047 \\
4 & 0.417 & 0.126 & 0.047 \\
5 & 0.474 & 0.199 & 0.068 \\
6 & 0.552 & 0.313 & 0.051 \\
7 & 0.424 & 0.241 & 0.057 \\
8 & 0.329 & 0.137 & 0.046 \\
9 & 0.675 & 0.320 & 0.047 \\
10 & 0.512 & 0.168 & 0.038 \\
11 & 0.294 & 0.082 & 0.019 \\
12 & 0.368 & 0.060 & 0.022 \\
13 & 0.532 & 0.198 & 0.131 \\
14 & 0.449 & 0.200 & 0.064 \\
15 & 0.283 & 0.145 & 0.038 \\
16 & 0.163 & 0.173 & 0.031 \\
Mean \pm standard & $0.428 \pm 0.127^{*}$ & $0.186 \pm 0.073^{\dagger}$ & $0.054 \pm 0.027^{\ddagger}$ \\
deviation & & & \\
\hline
\end{tabular}

LIF, leukemia inhibitory factor; group 1, normal group; group 2, moderate periodontitis group; group 3 , severe periodontitis group. ${ }^{*}$ Significant difference between group 1 and group $2(p<0.05)$. ${ }^{\dagger}$ Significant difference between group 2 and group $3(p<0.05)$. ${ }^{\ddagger}$ Significant difference between group 1 and group $3(p<0.05)$.
$<0.001)$, and group 1 and group $3(p<0.001)(p<0.05)$.

Table 5 demonstrates the correlations between gingival PMCA-1, IL-11, and LIF levels in normal gingiva and inflamed gingiva of chronic periodontitis. The correlations were given by Spearman correlation coefficient. There was positive relationship between PMCA-1 and IL-11 and relatively high correlation could be found. However, between PMCA-1 and LIF, as well as, IL-11 and LIF, negative rela-

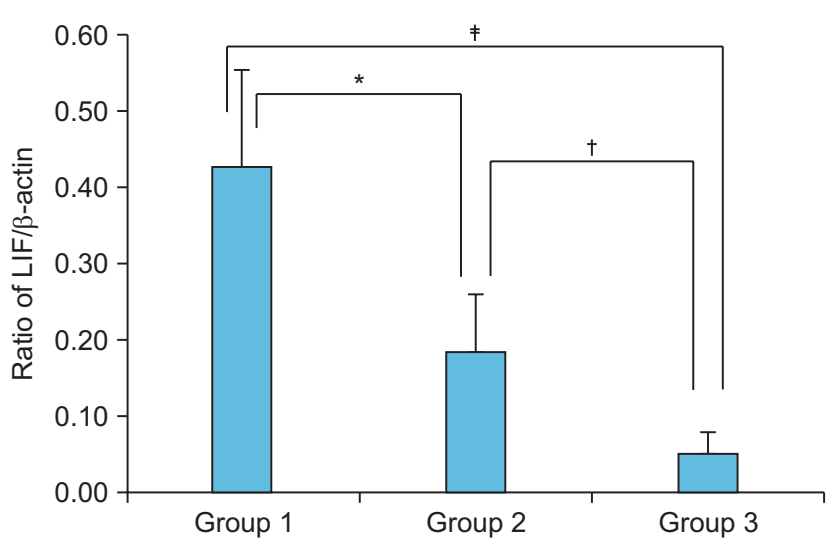

Fig. 6. Graph of normalized leukemia inhibitory factor (LIF) expression by LIF/ $\beta$-actin. The average amounts and standard deviation of LIF level (ratio of LIF/ $\beta$-actin) in group 1 (normal group), group 2 (moderate chronic periodontitis group), and group 3 (severe chronic periodontitis group) were used in this graph. The levels of LIF in the inflamed gingiva were higher than those of healthy gingiva. ${ }^{*}$ Significant difference between group 1 and group $2(p<0.001)$. ${ }^{\dagger}$ Significant difference between group 2 and group $3(p<0.001)$. ${ }^{\ddagger}$ Significant difference between group 1 and group $3(p<0.001)$.

Table 5. The correlations between gingival PMCA-1, IL-11, and LIF levels

\begin{tabular}{llll}
\multicolumn{1}{c}{ Variable } & PMCA-1 & IL-11 & LIF \\
\hline PMCA-1 & & & \\
$r$ & & $0.655^{*}$ & $-0.641^{*}$ \\
$p$-value & & $<0.001$ & $<0.001$ \\
$\quad$ number of samples & & 48 & 48 \\
IL-11 & & \\
$r$ & $0.655^{*}$ & & $-0.788^{*}$ \\
$p$-value & $<0.001$ & & $<0.001$ \\
number of samples & 48 & & 48 \\
LIF & & & \\
$r$ & $-0.641^{*}$ & $-0.788^{*}$ & \\
$p$-value & $<0.001$ & $<0.001$ & \\
number of samples & 48 & 48 & \\
\hline
\end{tabular}

PMCA, plasma membrane calcium ATPase; IL, interleukin; LIF, leukemia inhibitory factor; $r$, correlation coefficient.

${ }^{*}$ Statistically significant $(p<0.05)$. 
tionship could be observed and high correlation could be found between IL-11 and LIF.

\section{DISCUSSION}

Periodontitis is characterized as the disease with the host-mediated destruction of soft and hard tissue. Such destruction is occurred by induced production and activation of lytic enzymes along with stimulated osteoclastogenesis [20]. During this process, inflammatory mediators secreted from the host cells play important role and especially, bone resorption related factors, which is associated with osteoclast activity causing the resorption and destruction of alveolar bone, seems to have important role in the development and the process of periodontal disease.

There are only limited studies available focusing on the direct relation of PMCA-1 toward dental tissue though it is known to be related to the osteoclastogenesis and regulation of bone homeostasis [7]. Likewise, IL-11 and LIF which is well known inflammatory mediators yet has not been investigated to find out their correlation with periodontitis at the gingival tissue level in many studies. Therefore, in this study, we investigated expression pattern of PMCA-1, IL-11, and LIF, which are suggested to be possible boneresorption related factors, at the gingival tissue level in the different groups classified depending on the severity of periodontitis.

Generally, as chronic periodontitis progresses, osteoclastic activity becomes predominant [21]. From the result of this study, higher expression of PMCA-1 could be found as severity of chronic periodontitis aggravates. In other words, higher PMCA-1 expression could be seen as degree of alveolar bone resorption increases. This result can be explained by correlating it with the increased activity of bone-resorbing mature osteoclast. During the bone resorption process, excessive $\mathrm{Ca}^{2+}$ excreted from the bone goes into osteoclast. However, since such excessive amount of $\mathrm{Ca}^{2+}$ intake might be toxic to osteoclast, $\mathrm{Ca}^{2+}$ needs be continuously secreted to the extracellular space [22,23]. At this time, PMCA which is located at the basolateral membrane of mature osteoclast protects the osteoclast from the $\mathrm{Ca}^{2+}$ induced apoptosis by secreting $\mathrm{Ca}^{2+}$ toward outside of the cell [7]. However, considering the role of PMCA-1 which suppresses the osteo- clastogenesis and increased osteoclastogenesis in chronic periodontitis, it is difficult to relate it with our study result [24]. Thus, further studies on the exact action mechanism and effect of PMCA-1 seems necessary in near future.

Until now, studies investigating on the correlation between periodontitis and IL-11 only performed at the GCF level [11]. In this study based on the gingival tissue level, IL11 shows increased expression pattern as severity of periodontitis aggravates and differences in each group were all statistically significant. On the contrary, LIF shows highest level of expression in normal gingival tissue and expression level was decreased with statistical significance as severity of periodontitis aggravates. Above results are very similar with the result came from Becerik et al. [11]. They discovered that IL-11 GCF levels get increased in gingiva with periodontitis more than in normal gingiva, and reported their positive correlations with PD, CAL, and BOP. Meanwhile, in case of GCF LIF level, it was decreased in gingiva with periodontitis comparing to normal gingiva, and its negative correlations with PD and CAL were suggested. Based on limited results of this study, there is possibility that IL-11 might have a role as an inflammatory biomarker whereas LIF might have a beneficial role in the modulation of inflammatory response. If we could successfully utilize such factors with opposite characteristics in combination, it would be possible to accomplish more precise diagnosis and set up more effective treatment plan of periodontitis. Although this study results show relatively clear expression pattern of IL-11 and LIF as severity of periodontitis aggravates, it should be noted that there are still many conflicting results and only limited study available which directly observed amount of expression in gingival tissue level. Thus, further study on the expression pattern of IL-11 and LIF depending on the severity of periodontitis in GCF or gingival tissue level seems necessary.

Based on our study result synthesized with previous ones, it is clear that these factors all commonly affect the osteoclast formation. In addition, based on the high level of correlation, there is possibility that these factors might interact with each other in chronic periodontitis. However, evidence shows that IL-11 supports the osteoclast formation through RANKL-independent mechanism [25] whereas LIF stimulates bone resorption through stimulat- 
ing $\mathrm{Ca}^{2+}$ release and increasing RANKL mRNA expression [13]. Therefore, these cytokines are likely to affect the osteoclast formation with different mechanism. On the other hands, in case of PMCA-1, there is evidence that PMCA-1 suppresses the RANKL-induced $\mathrm{Ca}^{2+}$ oscillations and osteoclast differentiation [7]. Hence, there is possibility that LIF and PMCA-1 might affect each other through the pathway related to RANKL.

Considering the characteristic of periodontitis which tends to increase osteoclast formation, it would be probable result based on evidence up to present that expression of IL-11 and LIF should be increased while PMCA should be decreased in the aspect of osteoclast formation. However, since actual results of this study shows increased expression of PMCA-1 and IL-11 and decreased expression of LIF, there is gap between actual result and expected result. Such discrepancy might be due to characteristic of cytokine that functions can be varied depending on the target cell, cell environment/condition and the presence of other cytokine.

A forementioned 'dual role' of PMCA-1 would be easier to understand in the same context. Therefore, in order to understand the role and interaction of PMCA-1, IL-11, and LIF more properly, enhanced understanding on the role of individual factor would be necessary supported by further studies including comparative studies under controlled condition.

Periodontitis is inflammatory disease that accompanies alveolar bone resorption and destruction of periodontal connective tissue. Thus, it is important to understand the inflammatory destructive process of both soft and hard tissue and find out the related factors in this process for proper diagnosis and treatment of periodontitis. As inflammatory process of periodontal disease and role of regulatory factors toward it gets easier to understand through many studies, new strategies are being developed to prevent and treat periodontal disease. Factors subjected to this research were also studied for supporting such strategies.

In conclusion, PMCA-1, IL-11, and LIF seem to be associated with severity of chronic periodontitis and its existence, and these factors could affect each other during the progress of periodontitis. These factors are expected to be applied in the diagnosis and treatment of periodontitis through more comprehensive studies.

\section{CONFLICTS OF INTEREST}

The authors declare that they have no competing interests.

\section{ORCID}

\author{
Da-Le Yoon \\ https://orcid.org/0000-0003-4415-8689 \\ Su-Yeon Park \\ https://orcid.org/0000-0002-6431-5701 \\ Yong-Gun Kim \\ https://orcid.org/0000-0002-2793-7667 \\ Jae-Mok Lee \\ https://orcid.org/0000-0002-0291-6114
}

\section{REFERENCES}

1. Page RC, Offenbacher S, Schroeder HE, Seymour GJ, Kornman KS. Advances in the pathogenesis of periodontitis: summary of developments, clinical implications and future directions. Periodontol 2000 1997;14:216-248. doi: 10.1111/j.1600-0757.1997.tb00199.x.

2. Socransky SS, Haffajee AD. The bacterial etiology of destructive periodontal disease: current concepts. J Periodontol 1992;63(4 Suppl):322-331. doi: 10.1902/ jop.1992.63.4s.322.

3. Graves D. Cytokines that promote periodontal tissue destruction. J Periodontol 2008;79(8 Suppl):1585-1591. doi: 10.1902/jop.2008.080183.

4. Axelsen KB, Palmgren MG. Evolution of substrate specificities in the P-type ATPase superfamily. J Mol Evol 1998;46:84-101. doi: 10.1007/pl00006286.

5. Jensen TP, Filoteo AG, Knopfel T, Empson RM. Presynaptic plasma membrane $\mathrm{Ca} 2+$ ATPase isoform 2a regulates excitatory synaptic transmission in rat hippocampal CA3. J Physiol 2007;579(Pt 1):85-99. doi: 10.1113/jphysiol.2006.123901.

6. Talarico EF Jr, Kennedy BG, Marfurt CF, Loeffler KU, Mangini NJ. Expression and immunolocalization of plasma membrane calcium ATPase isoforms in human corneal epithelium. Mol Vis 2005;11:169-178.

7. Kim HJ, Prasad V, Hyung SW, Lee ZH, Lee SW, Bhargava A, Pearce D, Lee Y, Kim HH. Plasma membrane calcium ATPase regulates bone mass by fine-tuning osteoclast differentiation and survival. J Cell Biol 2012;199:1145-1158. doi: 10.1083/jcb.201204067.

8. Paul SR, Bennett F, Calvetti JA, Kelleher K, Wood CR, 
O'Hara RM Jr, Leary AC, Sibley B, Clark SC, Williams DA. Molecular cloning of a cDNA encoding interleukin 11, a stromal cell-derived lymphopoietic and hematopoietic cytokine. Proc Natl Acad Sci U S A 1990;87:7512-7516. doi: 10.1073/pnas.87.19.7512.

9. Kudo O, Sabokbar A, Pocock A, Itonaga I, Fujikawa Y, Athanasou NA. Interleukin-6 and interleukin-11 support human osteoclast formation by a RANKL-independent mechanism. Bone 2003;32:1-7. doi: 10.1016/s87563282(02)00915-8

10. Martuscelli G, Fiorellini JP, Crohin CC, Howell TH. The effect of interleukin-11 on the progression of ligature-induced periodontal disease in the beagle dog. J Periodontol 2000;71:573-578. doi: 10.1902/jop.2000.71.4.573.

11. Becerik S, Öztürk VÖ, Atmaca H, Atilla G, Emingil G. Gingival crevicular fluid and plasma acute-phase cytokine levels in different periodontal diseases. J Periodontol 2012;83:1304-1313. doi: 10.1902/jop.2012.110616.

12. Aghajanova L. Leukemia inhibitory factor and human embryo implantation. Ann N Y Acad Sci 2004;1034:176-183. doi: 10.1196/annals.1335.020.

13. Palmqvist P, Persson E, Conaway HH, Lerner UH. IL-6, leukemia inhibitory factor, and oncostatin $\mathrm{M}$ stimulate bone resorption and regulate the expression of receptor activator of NF-kappa B ligand, osteoprotegerin, and receptor activator of NF-kappa B in mouse calvariae. J Immunol 2002;169:3353-3362. doi: 10.4049/jimmunol.169.6.3353.

14. Xing L, Xiu Y, Boyce BF. Osteoclast fusion and regulation by RANKL-dependent and independent factors. World J Orthop 2012;3:212-222. doi: 10.5312/wjo.v3.i12.212.

15. Mühlemann HR, Son S. Gingival sulcus bleeding--a leading symptom in initial gingivitis. Helv Odontol Acta 1971;15:107-113.

16. Lindhe JT, Lang NP, Karring T. Clinical periodontology and implant dentistry. 5th ed. Ames: Blackwell Munksgaard; 2008;2:577.

17. Kim JB, Jung MH, Cho JY, Park JW, Suh JY, Lee JM. The influence of type 2 diabetes mellitus on the expression of inflammatory mediators and tissue inhibitor of metalloproteinases-2 in human chronic periodontitis. J Periodontal Implant Sci 2011;41:109-116. doi: 10.5051/ jpis.2011.41.3.109.

18. Jung HY, Kim YG, Park JW, Suh JY, Lee JM. The expression of a nitric oxide derivative, tissue inhibitors of metalloproteinase-3, and tissue inhibitors of metalloproteinase-4 in chronic periodontitis with type 2 diabetes mellitus. J Periodontal Implant Sci 2013;43:87-95. doi: 10.5051/ jpis.2013.43.2.87.

19. Cho JY, Xing S, Liu X, Buckwalter TL, Hwa L, Sferra TJ, Chiu IM, Jhiang SM. Expression and activity of human $\mathrm{Na}+$ /I- symporter in human glioma cells by adenovirusmediated gene delivery. Gene Ther 2000;7:740-749. doi: 10.1038/sj.gt.3301170.

20. Graves DT, Cochran D. The contribution of interleukin-1 and tumor necrosis factor to periodontal tissue destruction. J Periodontol 2003;74:391-401. doi: 10.1902/ jop.2003.74.3.391.

21. Wiebe SH, Hafezi M, Sandhu HS, Sims SM, Dixon SJ. Osteoclast activation in inflammatory periodontal diseases. Oral Dis 1996:2:167-180. doi: 10.1111/j.1601-0825.1996. tb00218.x.

22. Di Leva F, Domi T, Fedrizzi L, Lim D, Carafoli E. The plasma membrane $\mathrm{Ca}^{2+}$ ATPase of animal cells: structure, function and regulation. Arch Biochem Biophys 2008;476:65-74. doi: 10.1016/j.abb.2008.02.026.

23. Moonga BS, Li S, Iqbal J, Davidson R, Shankar VS, Bevis PJ, Inzerillo A, Abe E, Huang CL, Zaidi M. Ca(2+) influx through the osteoclastic plasma membrane ryanodine receptor. Am J Physiol Renal Physiol 2002;282:F921-F932. doi: 10.1152/ajprenal.00045.2000.

24. Taubman MA, Valverde P, Han X, Kawai T. Immune response: the key to bone resorption in periodontal disease. J Periodontol 2005;76(11 Suppl):2033-2041. doi: 10.1902/ jop.2005.76.11-S.2033.

25. Girasole G, Passeri G, Jilka RL, Manolagas SC. Interleukin-11: a new cytokine critical for osteoclast development. J Clin Invest 1994;93:1516-1524. doi: 10.1172/JCI117130. 